Vol. 14, No. 4, December 2010, pp. 290-297

Invited Paper

\title{
Planar Waveguide Devices for Communication and Sensing Applications
}

\author{
Katsunari Okamoto* \\ AiDi Corporation, Tsukuba, Ibaraki 305-0032, Japan
}

(Received October 19, 2010 : revised November 15, 2010 : accepted November 15, 2010)

\begin{abstract}
The paper reviews progress and future prospects of two kinds of planar waveguide devices; they are (a) silica and silicon photonics multi/demultiplexers for communications and signal processing applications, and (b) a novel waveguide spectrometer based on Fourier transform spectroscopy for sensing applications.
\end{abstract}

Keywords: Silica waveguide, Silicon photonics, AWG, Echelle grating, Fourier transform spectroscopy OCIS codes : (060.4510) Optical communications; (230.3120) Integrated optics devices; (300.6300) Spectroscopy, Fourier transforms

\section{INTRODUCTION}

Silicon photonics is widely regarded as a promising technology to meet the requirements of rapid bandwidth growth and energy-efficient on-chip communication while reducing cost per bit. In all potential application areas it is widely recognized that use of wavelength division multiplexing (WDM) techniques will be critical in achieving the required high levels of data transmission. Si photonics devices will have to deal with several tens of different wavelengths of light in the next-generation multi-core CMOS chips.

There are mainly four kinds of devices capable of multi/ demultiplexing tens of WDM signals; they are ring resonators, lattice-form filters, arrayed waveguide gratings (AWG) and planar Echelle gratings. The former two are cascaded devices relying on temporal multi-beam interference effect and the latter two utilize spatial multi-beam interference effect. In order to achieve good crosstalk characteristics in the temporal and spatial multi-beam interference effects, uniformity of effective index $n_{c}(=\beta / k)$, where $\beta$ and $k$ denote propagation constant and wave number, is critically important. Filter characteristics of four kinds of devices will be investigated and performance limitations of silicon photonics filters are discussed.

In the latter part of the paper, an integrated-optic spectrometer based on Fourier-transform spectroscopy will be described. A novel planar waveguide spectrometer consists of interleaved Mach-Zehnder interferometer (MZI) array. Practical importance of Fourier-transform spectrometer is the ability to correct for interferometer defects caused by effective index fluctuations (phase errors) in data processing stage. Successful measurement results of the signal spectrum with $20-\mathrm{GHz}$ resolution by the spectrometer implemented in silica-based planar waveguide will be presented.

\section{SILICON PHOTONICS WDM FILTERs}

Flat-top passband characteristics are required for filters in most of the WDM applications [1-4]. Crosstalk degradation in coupled ring resonators and lattice-form filters are caused by both gap width error in directional couplers and optical path length error in the waveguides.

Effective-index fluctuation $\delta n_{c}$ in silica Planar Lightwave Circuits (PLCs) have been measured to be about $\delta n_{c} \sim 1 \times 10^{-6}$ by using Fourier transform spectroscopy [5]. Good crosstalk characteristics are obtained in silica-based AWGs as shown in Fig. 1. The relation between the effective-index fluctuation $\delta n_{c}$ and crosstalk (XT) has been investigated theoretically and experimentally [6]. Based on the investigations, the empirical expression for the relation between $\delta n_{c}$ and XT has been obtained as

$$
\mathrm{XT} \sim 10 \log \left(\frac{\delta \mathrm{n}_{\mathrm{c}} \mathrm{L}_{\mathrm{ctr}}}{\lambda}\right)^{2},
$$

where $L_{c t r}$ and $\lambda$ denote array waveguide length in the central region and the wavelength of light signal, respectively. $L_{c t r}$ of AWG in Fig. 1 is $L_{c t r} \sim 15 \mathrm{~mm}$. Substituting

\footnotetext{
*Corresponding author: katsu.okamoto@aidicorp.com

Color versions of one or more of the figures in this paper are available online.
} 


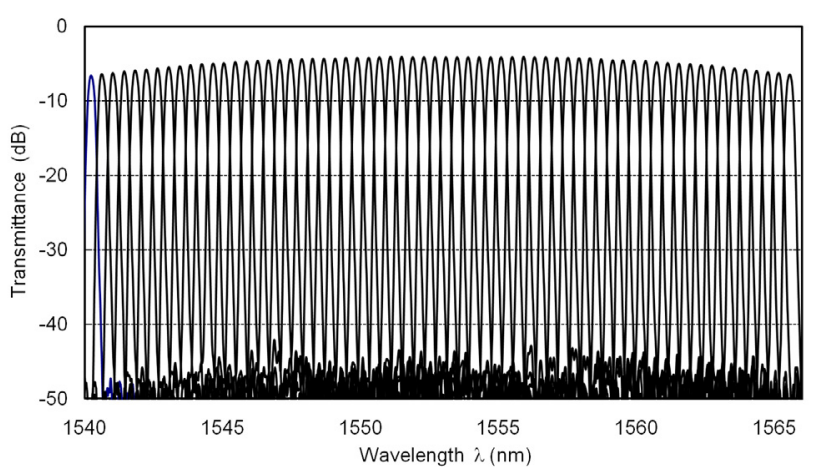

FIG. 1. Demultiplexing properties of $64 \mathrm{ch}-50 \mathrm{GHz}$ spacing silica AWG.

$\delta n_{c} \sim 1 \times 10^{-6}, L_{c t r} \sim 15 \mathrm{~mm}$, and $\lambda=1.55 \mu \mathrm{m}$ into Eq. (1), we obtain XT $\sim-40 \mathrm{~dB}$. Although Eq. (1) gives good agreement with the experimental results, the author believes that it should be proved analytically. Crosstalk of the planar Echelle gratings is obtained based on the similar investigations as

$$
\mathrm{XT} \sim 10 \log \left(\frac{\mathrm{n}_{\mathrm{s}} 2 \delta \zeta}{\lambda}\right)^{2}
$$

where $n_{s}$ and $\delta \zeta$ denote the effective index in the slab region and the facet position fluctuation in the reflection mirrors. It is known from Eq. (2) that $\delta \zeta$ should be about $5 \mathrm{~nm}$ in order to obtain $-40 \mathrm{~dB}$ crosstalk in silica Echelle grating $\left(n_{s} \sim 1.5\right)$. Such a small $\delta \zeta$ would be possible in the laboratory experiments [7]. However, the mask resolution for the commercial PLC products is normally about $25 \mathrm{~nm}$ $\sim 50 \mathrm{~nm}$. This is the reason why commercial planar Echelle gratings could not achieve sufficient crosstalk value to be used in the current WDM systems.

Effective-index fluctuation $\delta n_{c}$ in Si-wire waveguides has been evaluated from the measurements on the variation of resonant wavelength in the cascaded ring resonators [8]. Peak-to-peak resonance wavelength variation is $2 \delta \lambda \sim 2.4 \mathrm{~nm}$. Then, $\delta n_{c}$ is obtained as $\delta n_{c}=n_{c} \delta \lambda / \lambda_{0} \sim 2 \times 10^{-3}$, where $n_{c}=2.519$ and $\lambda_{0}=1.55 \mu \mathrm{m}$. Effective-index fluctuation $\delta n_{c}$ in Si-wire waveguides can also be calculated by the vectorial finite element method [3]. Fig. 2(a) shows $n_{c}$ of Si-wire waveguide with thickness $2 \mathrm{t}=0.22 \mu \mathrm{m}$ (blue) and its dependence on the core width $2 \mathrm{a}(\mathrm{red}) . d n_{c} / d(2 a)$ at the typical core width $2 \mathrm{a}=0.5 \mu \mathrm{m}$ is $1.3 \mu \mathrm{m}^{-1}=1.3 \times 10^{-3} \mathrm{~nm}^{-1}$. Fig. 2(b) shows $n_{c}$ of Si-wire waveguide with width $2 \mathrm{a}=$ $0.5 \mu \mathrm{m}$ (blue) and its dependence on the core thickness $2 \mathrm{t}$ (red). $d n_{c} / d(2 t)$ at the typical core thickness $2 \mathrm{t}=0.22 \mu \mathrm{m}$ is $3.4 \mu \mathrm{m}^{-1}=3.4 \times 10^{-3} \mathrm{~nm}^{-1}$.

Core side-wall roughness of Si-wire waveguide is measured to be about $\sigma_{\text {width }} \sim 2 \mathrm{~nm}$ [9]. Top silicon thickness uniformity of 6-inch SOI (silicon on insulator) wafer is measured to be about $+/-2 \mathrm{~nm}$ [10]. However, the photonic device occupies just $\sim 1 \mathrm{~mm}$ square or less.

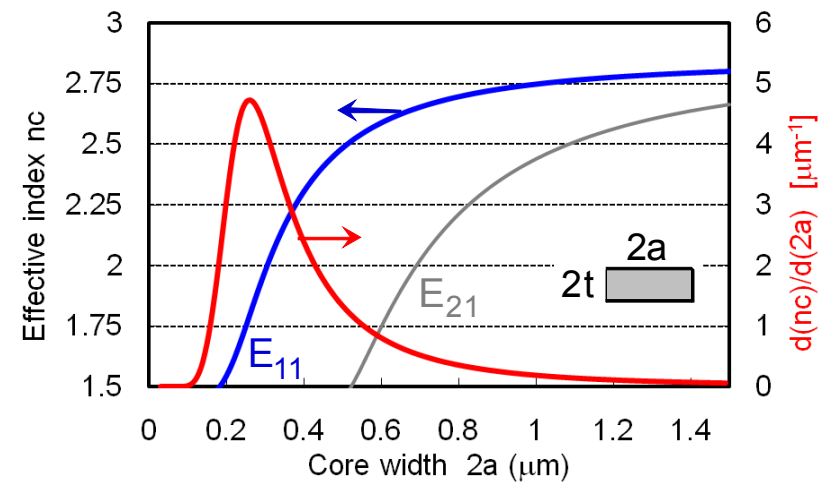

(a)

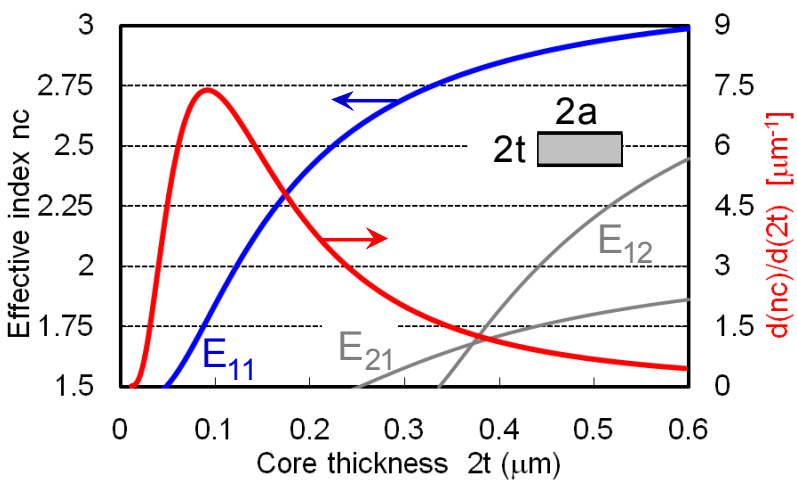

(b)

FIG. 2. (a) Effective index $n_{c}$ of Si-wire waveguide with $0.22 \mu \mathrm{m}$ thickness (blue) and its dependence on the core width $2 \mathrm{a}$ (red). (b). Effective index $n_{c}$ of Si-wire waveguide with $0.5 \mu \mathrm{m}$ width (blue) and its dependence on the core width $2 \mathrm{t}$ (red).

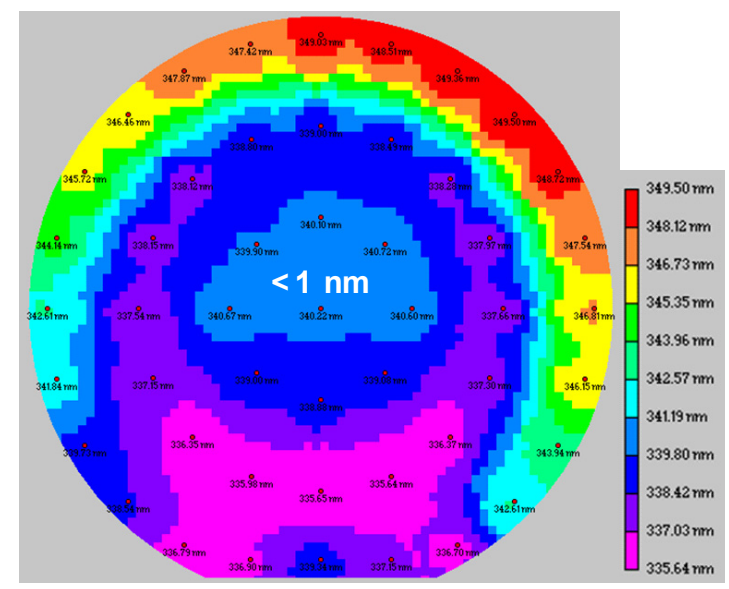

FIG. 3. Thickness variation in 6-inch SOI wafer.

Then, the thickness fluctuation would be less than $\sigma_{\text {thickness }}$ $\sim 1 \mathrm{~nm}$ as shown in the central region of Fig. 3 [6]. Effective-index fluctuation $\delta n_{c}$ in Si-wire waveguide is then estimated to be $\delta n_{c}=\left[d n_{c} / d(2 a)\right] \times \sigma_{\text {width }} \sim 2.6 \times 10^{-3}$, or $\delta n_{c}=\left[d n_{c} / d(2 t)\right] \times \sigma_{\text {thickness }} \sim 3.4 \times 10^{-3}$, respectively. Both of the theoretical estimations for the effective-index fluctuation $\delta n_{c}$ agree well with the measured value. Effective- 
index fluctuation $\delta n_{c}$ against core width variation can be reduced by enlarging core width in the straight regions. For example, $d n_{c} / d(2 a)$ becomes to $0.35 \mu \mathrm{m}^{-1}=$ $3.5 \times 10^{-4} \mathrm{~nm}^{-1}$ at $2 \mathrm{a}=0.8 \mu \mathrm{m}$ [11] as shown in Fig. 2(a).

Si-rib waveguide structure allows us to obtain much smaller $\delta n_{c}$ as shown in Fig. 4, where core width W, core height $\mathrm{H}$, and etch depth $\mathrm{h}$ are $\mathrm{W}=0.5 \mu \mathrm{m}, \mathrm{H}=0.3 \mu \mathrm{m}$, and $\mathrm{h}=0.15 \mu \mathrm{m}$, respectively. $d n_{c} / d W$ at $\mathrm{W}=0.5 \mu \mathrm{m}$ is $6.4 \times 10^{-4} \mathrm{~nm}^{-1}$. However, the minimum bending radius becomes much larger compared to $\mathrm{Si}$-wire waveguides and makes the device size larger. Therefore, Si-wire waveguide is still very attractive in making compact photonic devices.

Effective-index fluctuations of the order of $\delta n_{c}=3 \times 10^{-4}$ $\sim 2 \times 10^{-3}$ determine the crosstalk values of the current Si-wire AWGs to be about $-20 \mathrm{~dB}$ [11-13].

Echelle grating has been believed to be advantageous over AWG because only the facet position error causes crosstalk degradation. But, this is not true in $\mathrm{Si}$ Echelle grating since the effective-index fluctuation $\delta n_{s}$ in the slab waveguide is substantially large as shown in Fig. 5 . $d n_{s} / d(2 t)$ at the typical core thickness $2 \mathrm{t}=0.22 \mu \mathrm{m}$ is $3.3 \times 10^{-3} \mathrm{~nm}^{-1}$. Then, Eq. (2) for Si Echelle grating should be rewritten as

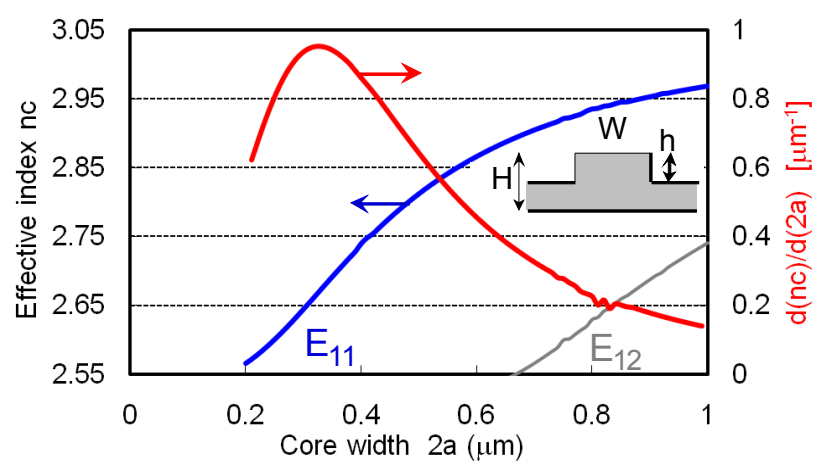

FIG. 4. Effective index $n_{c}$ of Si-rib waveguide (blue) and its dependence on the core width $\mathrm{W}$ (red).

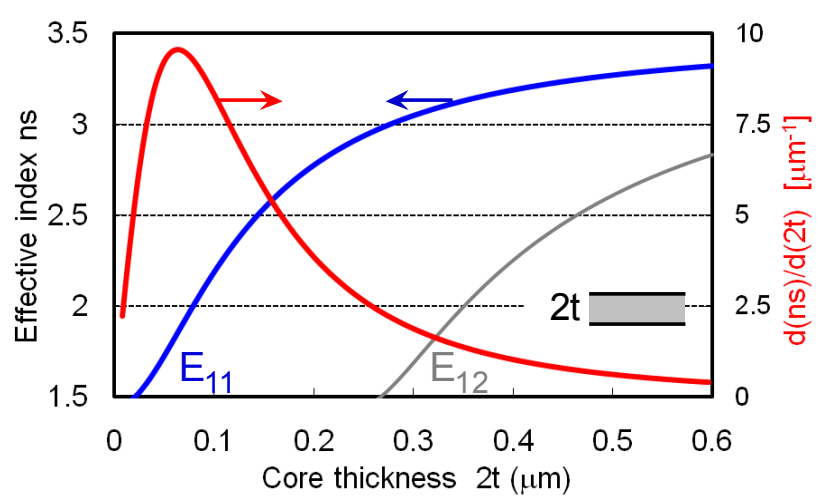

FIG. 5. Effective index $n_{s}$ of Si slab waveguide (blue) and its dependence on the core width $2 t$ (red).

$$
\mathrm{XT} \sim 10 \log \left(\left|\frac{\mathrm{n}_{\mathrm{s}} 2 \delta \zeta}{\lambda}\right|+\left|\frac{\delta \mathrm{n}_{\mathrm{s}} \mathrm{L}_{\mathrm{ctr}}}{\lambda}\right|\right)^{2},
$$

where $L_{c t r}$ denotes the total path length in the central region of the slab. Eq. (3) explains why large channel count (30 ch-3.2 nm spacing) Echelle grating has about $-15 \mathrm{~dB}$ crosstalk, though small channel count (4 ch-20 nm spacing) one had about $-30 \mathrm{~dB}$ crosstalk $[14,15]$. The total path length $L_{c t r}$ in the large channel count Echelle grating is almost 5 times longer than that of small channel count one. Therefore, the second term in Eq. (3) becomes dominant in the large channel count Echelle grating.

In order to achieve good crosstalk characteristics $(<-20$ $\mathrm{dB}$ ) in both Si-wire AWG and Echelle grating, (a) improvement in core width and thickness uniformity and/or (b) size reduction to make $L_{c t r}$ small are strongly required.

Cascaded ring resonators and lattice-form filters are also attractive candidates for filter applications in Si photonics. Fig. 6 shows coupled resonator optical waveguide (CROW) [1]. Kappa's are amplitude coupling coefficients in the directional couplers. Ring perimeter $\mathrm{L}=27.7 \mu \mathrm{m}$ for the ring diameter of $4.4 \mu \mathrm{m}$. Free spectral range (FSR) between the adjacent resonance order is about $2,600 \mathrm{GHz}$, which allows us to make $13 \mathrm{ch}-200 \mathrm{GHz}(1.6 \mathrm{~nm})$ spacing WDM filter. FSR is inversely proportional to $\mathrm{L}$. Therefore, ring resonator using other type of waveguide (such as silica PLC with bend radius of $5 \mathrm{~mm}$ ) cannot obtain sufficiently wide FSR. Fig. 7(a) shows a filter response without effective index fluctuation (dotted lines) and with index fluctuation $\delta n_{c}=5 \times 10^{-4}$ only in the third ring (solid lines). It is shown that $\delta n_{c}$ less than $10^{-3}$ degrades crosstalk characteristics drastically. Fig. 7(b) is a filter response when $\kappa_{0}$ is deviated from its ideal value 0.47 to 0.42 . This amount of $\kappa_{0}$ deviation can be caused by about $+/-13 \mathrm{~nm}$ gap width variation in the directional coupler.

Fig. 8 shows schematic configuration of three-stage latticeform filter [2]. $c_{1}$ to $c_{4}$ are power coupling coefficients in the directional couplers. Channel spacing between the two

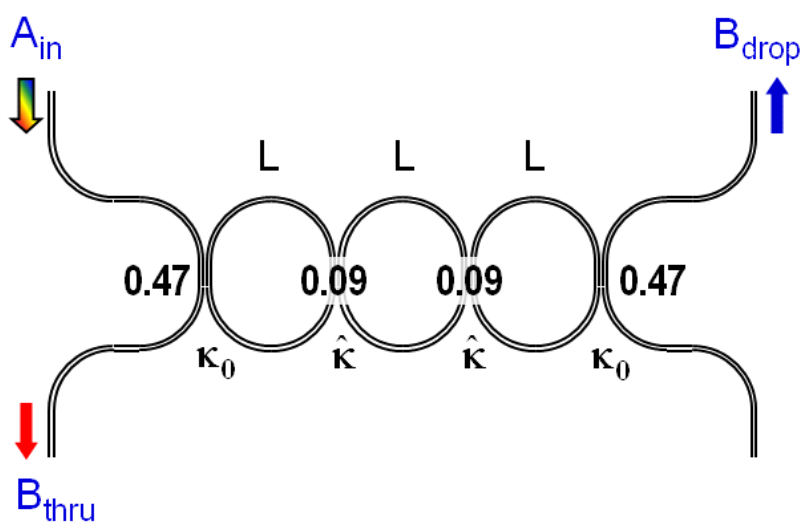

FIG. 6. Coupled resonator optical waveguide (CROW). 


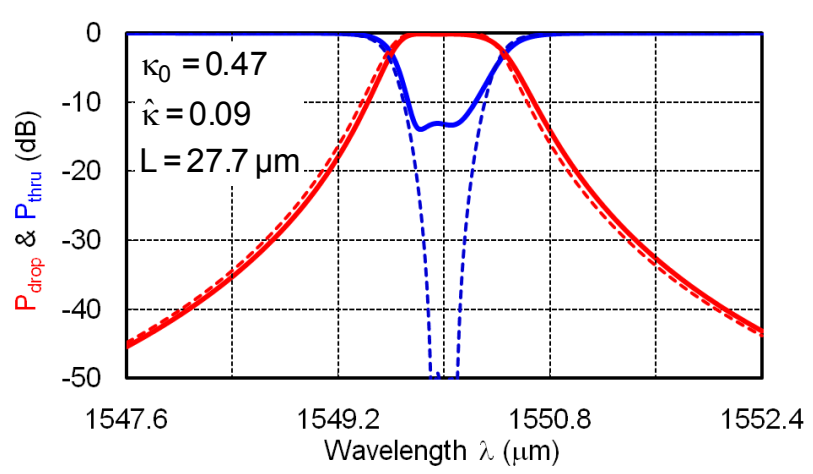

(a)

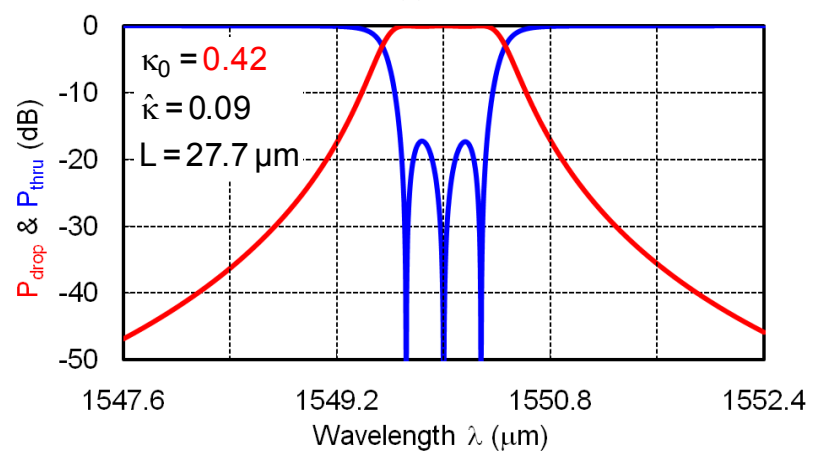

(b)

FIG. 7. (a) Filter response without effective index fluctuation (dotted lines) and $\delta \mathrm{n}_{\mathrm{c}}=5 \times 10^{-4}$ only in the third ring (solid lines). (b). Filter response when $\kappa_{0}$ is deviated from 0.47 to 0.42 .

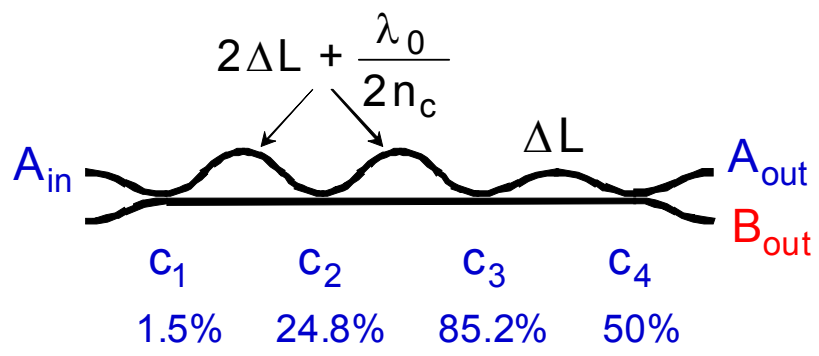

FIG. 8. Three-stage lattice-form filter.

output ports is given by

$$
\mathrm{S}_{\mathrm{ch}}=\frac{\mathrm{c}}{2 \mathrm{~N}_{\mathrm{c}} \Delta \mathrm{L}}
$$

where $\Delta L, N_{c}$ and $\mathrm{c}$ are path length difference, group index $\left(N_{c}=n_{c}-\lambda d n_{c} / d \lambda\right)$ of the waveguide and light velocity. In the typical Si-wire waveguide, $\Delta L=93 \mu \mathrm{m}$ gives the channel spacing $S_{c h}=400 \mathrm{GHz}$. Fig. 9 shows a filter response without effective index fluctuation (dotted lines) and with random index fluctuation $\left|\delta n_{c}\right|<5 \times 10^{-4}$ (solid lines). It is shown that $\delta n_{c}$ less than $10^{-3}$ degrades crosstalk characteristics drastically. It is also shown by other numerical simulation that when $c_{2}$ is deviated from

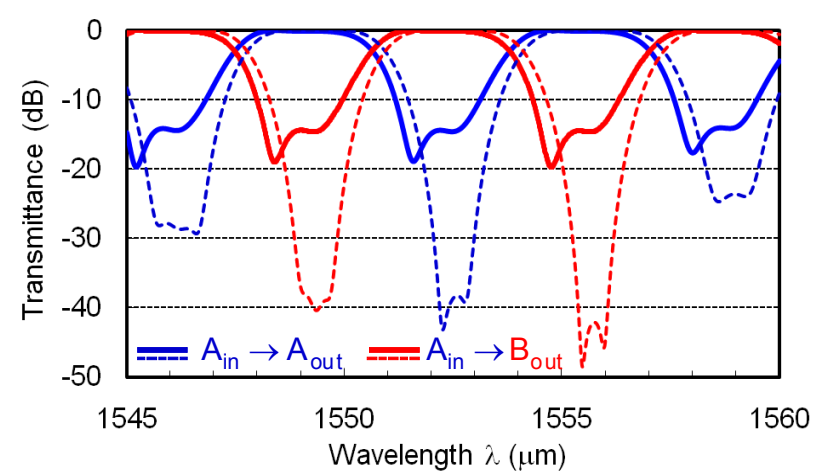

FIG. 9. Filter response without effective index fluctuation (dotted lines) and random index fluctuation $\left|\delta \mathrm{n}_{\mathrm{c}}\right|<5 \times 10^{-4}$ (solid lines).

its ideal value $24.8 \%$ to $17.8 \%$, crosstalk becomes about $-20 \mathrm{~dB}$. This amount of $\mathrm{c}_{2}$ deviation can be caused by about $+/-20 \mathrm{~nm}$ gap width variation in the directional coupler. Experimentally, crosstalk level of about $-10 \mathrm{~dB}$ has been obtained in Si-wire lattice-form filters [16].

\section{PLANAR FT SPECTROMETER}

Spatial heterodyne spectroscopy (SHS) is an interferometric Fourier-transform technique based on a modified Michelson interferometer with no moving parts and relying on analysis of stationary interference patterns [17]. In the bulk-optic SHS, the mirrors of the Michelson interferometer are replaced by diffraction gratings to offer scanning capability for two wavefronts. The SHS concept has been generalized into a waveguide Mach-Zehnder interferometer (MZI) array based on cosine fast Fourier transform (FFT) [18]. The path length difference in each asymmetrical MZI increases by $\Delta L$, similar to AWG [3]. For any input wavelength, the entire output of a MZI array creates spatially oscillatory patterns, where the oscillation period is wavelength dependent due to the dispersive nature of the asymmetrical MZI. Each input wavelength has a unique fringe representation, which is the basis of the Fourier-transform relation between the input spectra and the output interferogram. Employing multiple input waveguides in the waveguide SHS is an important advantage over the existing AWG-type spectrometer, since it greatly increases the light capturing capability in proportion to the number of MZIs by using, for example, photonic lantern technology [19]. A photonic lantern is a device that efficiently converts light from a multimode fiber tip for light capturing to single-mode fibers connected to MZI input waveguides. The practical importance of the Fourier-transform spectrometer is the ability to correct for interferometer defects (phase errors) in data processing stage. The first waveguide SHS have been fabricated in silicon-on-insulator rib waveguides using stacks of multiple MZIs [20]. However, a measurement result on the spectrum 
has not been reported so far to our knowledge.

We reported a novel planar waveguide SHS configuration with an interleaved MZI array [21]. The interleaved configuration allows us to put 2 3 times more MZIs than the simple stacking layout when we compare two layout methods for the same spectrometer parameters. We made an array of 32 MZIs in silica-based PLC. The path length difference increment was $\Delta L=162 \mu \mathrm{m}$, which resulted in $20 \mathrm{GHz}$ resolution at the $1.55 \mu \mathrm{m}$ region. Phase errors in the asymmetrical MZIs were measured by the thermo-optic phase scanning method [22]. The spectrum of the light source was successfully retrieved by correcting the MZI output data with the phase error values.

Figure 10 shows the configuration of the novel waveguide SHS with the interleaved MZI array. The total number of MZIs is $\mathrm{N}=32$. The waveguide core size is $4.5 \mu \mathrm{m} \times 4.5 \mu \mathrm{m}$ with $1.5 \%$ refractive-index difference. The minimum bend radius is $2 \mathrm{~mm}$. White boxes indicate $3 \mathrm{~dB}$ couplers consisting of either directional couplers or multimode interference couplers. The input and output waveguide separations at the chip ends are 15 and $50 \mu \mathrm{m}$, respectively. Waveguide arms in the MZI are intentionally inclined to both sides so that the waveguides intersect by more than $45^{\circ}$ with each other. It is known that the excess loss of the waveguide crossing can be reduced as low as $\sim 0.02 \mathrm{~dB} /$ intersection when crossing angle is larger than $45^{\circ}$ [23]. Dummy crossing waveguides are placed to make the total number of waveguide crossing equal for all MZIs. The chip size, including input and output fan-out regions, is $43 \mathrm{~mm} \times 25 \mathrm{~mm}$. We measured both cross-port and through-port outputs $p(k)$ and $q(k)$ in the $k$-th $(\mathrm{k}=0$ $\sim \mathrm{N}-1)$ MZI so that the spatial nonuniformity of the input light distribution can be corrected. The output power of each port was measured by shifting the single-mode fiber in the current preliminary experiments. Fiber-to-fiber loss of MZIs are measured to be $4.2 \sim 5.4 \mathrm{~dB}$.

The operational principle of a SHS spectrometer based on the MZI array has been elaborated in [18]. For a signal $s(f)$ passing through the $k$-th MZI, a normalized cross-port output is given by assuming negligible waveguide loss as

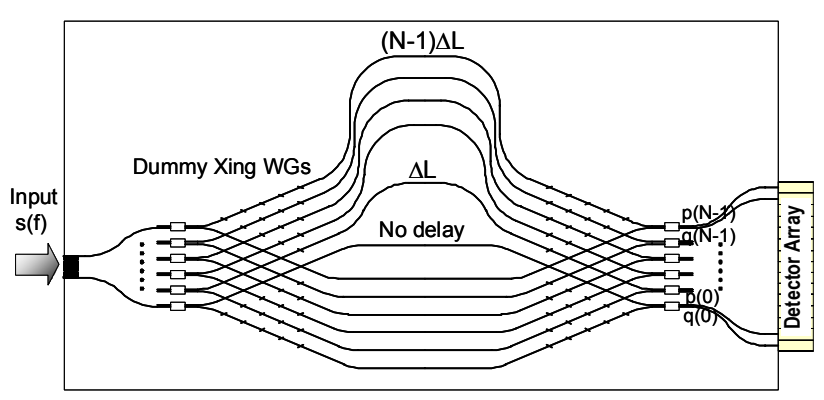

FIG. 10. Planar waveguide SHS configuration with interleaved MZI array.

$$
\mathrm{P}(\mathrm{k})=\frac{\mathrm{p}(\mathrm{k})}{\mathrm{p}(\mathrm{k})+\mathrm{q}(\mathrm{k})}=\frac{1}{\mathrm{~S}} \int_{\mathrm{f}_{0}}^{\mathrm{f}_{\mathrm{f}}+\mathrm{SSR}} \mathrm{s}(\mathrm{f}) \frac{[1+\cos (\beta \mathrm{k} \Delta \mathrm{L})]}{2} \mathrm{df} \quad(\mathrm{k}=0 \sim \mathrm{N}-1),
$$

where $\beta$ is a propagation constant, FSR is a free spectral range determined by $\Delta L$, and $S=\int_{f_{0}}^{f_{0}+F S R} s(f) d f . f_{0}$ is denoted as the Littrow frequency at which phase delays in different MZIs become integer multiples of $2 \pi$ $\left(\beta\left(f_{0}\right) \Delta L=2 m \pi\right)$. Since MZI response repeats periodically with FSR, one needs to block the unnecessary spectral range by a bandpass filter. Based on the discrete cosine Fourier transform, the input spectrum $s\left(f_{n}\right)\left(f_{n}=f_{0}+n \cdot F S R / \hat{N}\right.$, where $\hat{N}=2 N=64$ ) can be calculated from the measured output power $P(k)$ as

$$
\mathrm{s}\left(\mathrm{f}_{\mathrm{n}}\right)=\mathrm{A} \sum_{\mathrm{k}=0}^{\hat{\mathrm{N}}-1} \mathrm{P}(\mathrm{k}) \cos \left(2 \pi \frac{\mathrm{nk}}{\hat{\mathrm{N}}}\right) \quad(\mathrm{n}=0 \sim \mathrm{N}-1) .
$$

In the above equation, $A$ is a constant and $P(k)$ for $n=N \sim \hat{N}-1$ is assumed to be $P(\hat{N}-k)$. Although FSR was sampled with $\mathrm{N}$ points in [18], we sample it with $2 \mathrm{~N}$ points in order to correctly represent the MZI outputs. Because MZI responses for the signal in the upper half of FSR, $s\left(f_{n}\right) \quad(n=N \sim \hat{N}-1)$, have identical spatial fringe representation to those of the signal in the lower half, only the lower half of the signal spectrum can be measured. Resolution of the spectrometer is given by $\delta f=c /\left(\hat{N} n_{c} \Delta L\right)=20 \mathrm{GHz}$. Phase errors caused by effectiveindex fluctuations in the MZI array deteriorate the accuracy in the retrieved signal by Eq. (6). Phase error $\delta \phi_{k}$ in the $k$-th MZI is expressed as $\delta \phi_{k}=\left(2 \pi / \lambda_{0}\right) \delta n_{c}(k) L_{k}$, where $\delta n_{c}(k)$ and $L_{k}$ denote effective-index fluctuation and MZI arm length, as shown in Fig. 11(a). A heater with length $\ell$ was placed from outside of the chip on either one of the MZI arms to measure $\delta \phi_{k}$. The through-port transmittance $q(k)$ under the thermo-optic effect is given by

$$
\mathrm{q}(\mathrm{k})=\frac{1}{2}\left\{1-\cos \frac{2 \pi}{\lambda_{0}}\left[\alpha \mathrm{H} \ell-\delta \mathrm{n}_{\mathrm{c}}(\mathrm{k}) \mathrm{L}_{\mathrm{k}}\right]\right\} .
$$

Here, $H$ is a heater power applied to the phase shifter, $\alpha$ is a coefficient of thermo-optic refractive-index change per unit heater power and $\lambda_{0}=c / f_{0}$. Figure 11(b) shows an example of the thermo-optic phase scanning measurement. The first extinction point indicated by $H_{0}$ corresponds to the point at which the phase error is compensated for. The power between two adjacent extinction points $H_{T}$ corresponds to an optical path length change with $\lambda_{0}$. $\delta \phi_{k}$ is then given by $\delta \phi_{k}=2 \pi \cdot H_{0} / H_{T}$. Effective-index fluctuation is obtained as $\delta n_{c}(k)=\left(\delta \phi_{k} / L_{k}\right) \lambda_{0} / 2 \pi$. Measured 


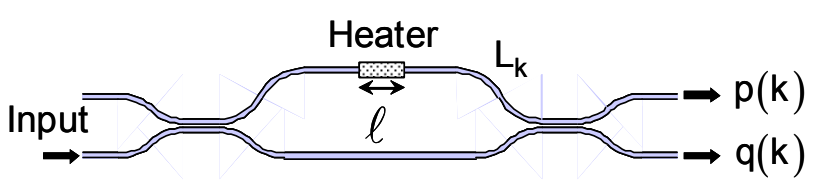

(a)

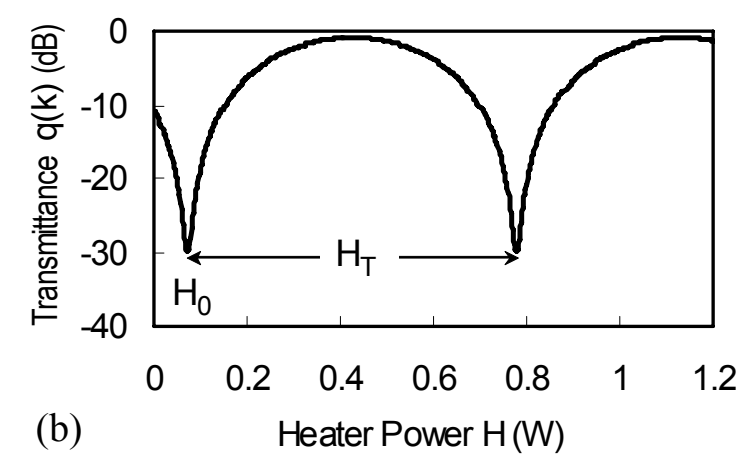

FIG. 11. (a) Mach-Zehnder interferometer in the $k$ th stage and (b) through-port response versus heater power.

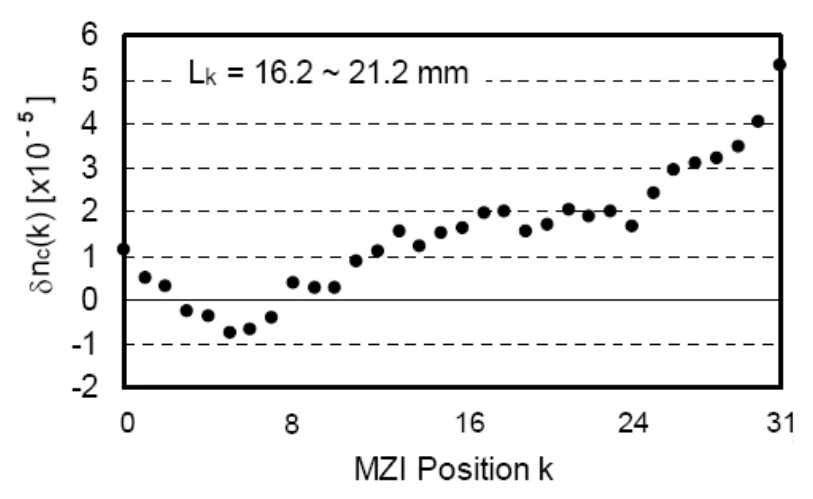

FIG. 12. Measured effective-index fluctuation in the MZI array.

$\delta n_{c}(k)$ in the MZI array is shown in Fig. 12.

A major advantage of the waveguide SHS based on Fourier-transform spectroscopy is that the interferometer defects due to effective-index fluctuations can be corrected in data processing stage. On the contrary, physical refractiveindex correction has been required for AWGs after the phase error measurement [24]. A known signal spectrum was coupled into the waveguide SHS. The normalized cross-port output power distribution $P(k)$ and the retrieved signal spectrum $s\left(\lambda_{n}\right)\left(\lambda_{n}=c / f_{n}\right)$ by the cosine FFT are shown in Figs. 13 and 14, respectively. The Littrow wavelength $\lambda_{0}$ was determined by measuring the normalized cross-port output at the second MZI $P(k=1)$. $P(1)$ rapidly oscillates with respect to the wavelength change. But, it reaches to stationary and the maximum value at $\lambda_{\max }=\lambda_{0}\left[1+\delta \phi_{1} /(2 \pi m)\right] \sim \lambda_{0} . \quad \lambda_{0}$ was measured to be $1550.1 \mathrm{~nm}$. Solid curve in Fig. 14 is the original signal spectrum measured by a bulk spectrometer. The Hanning

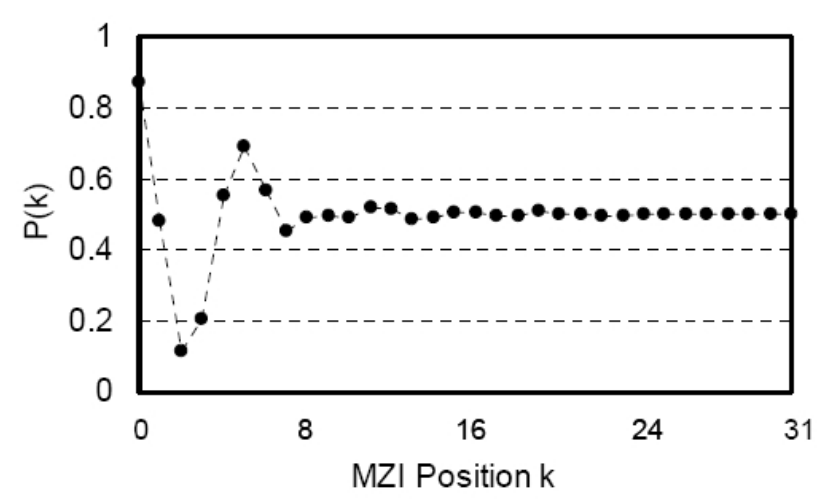

FIG. 13. Measured cross-port output power distribution in the MZI array.

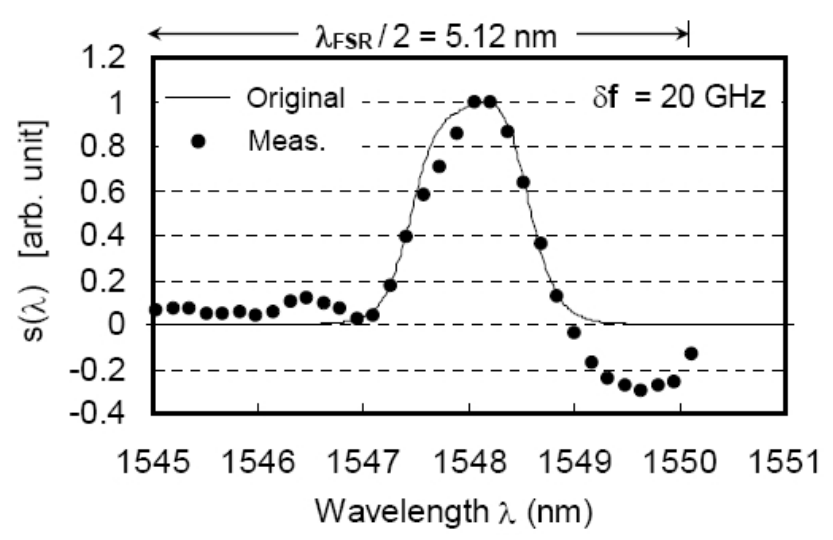

FIG. 14. Retrieved signal spectrum by cosine Fourier transform.

window was multiplied to $P(k)$ so as to reduce oscillatory features in the retrieved spectrum. The FSR in terms of wavelength is given by $\lambda_{F S R}=\lambda_{0} / m=10.24 \mathrm{~nm}$. Signals in the range of $\lambda=1539.9 \sim 1545.04 \mathrm{~nm}$ are not shown in Fig. 14 since they are mirrored replica of the spectrum in $\lambda=1545.2 \sim 1550.1 \mathrm{~nm}$. Deviation of the measured spectrum from the original one is mainly due to the phase error $\delta \phi_{k}^{\prime} s$ since they cannot be directly included in the cosine FFT analysis. The horizontal axis in Fig. 13 is understood to be the optical path length difference divided by $n_{c} \Delta L$. Then, the measured MZI output should be located at $k+\delta n_{c} L_{k} / n_{c} \Delta L$ instead of the ideal position, $k$. The correction can be done by using the measured data and the Lagrange interpolation to replace the unevenly spaced interferogram points by the evenly spaced ones [25]. Fourier-transform algorithms can then be applied to the evenly spaced interferogram points. For a large number of data points, as in bulk-optic Fourier-transform spectroscopy, a FFT-based procedure is a prerequisite. However, for a small number of $\mathrm{N}$, the discretized form of Eq. (1), including phase errors,

$$
\mathrm{P}(\mathrm{k})=\frac{1}{\mathrm{~S}} \sum_{\mathrm{n}=0}^{\mathrm{N}-1} \frac{\mathrm{s}\left(\mathrm{f}_{\mathrm{n}}\right)}{2}\left[1+\cos \left(2 \pi \frac{\mathrm{nk}}{\hat{\mathrm{N}}}+\delta \varphi_{\mathrm{k}}\right)\right] \quad(\mathrm{k}=0 \sim \mathrm{N}-1),
$$




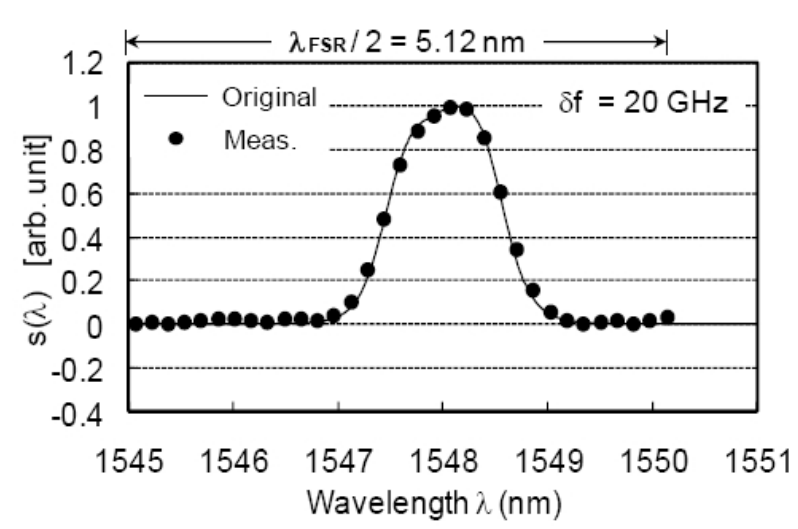

FIG. 15. Signal spectrum corrected by taking account of the measured phase errors.

can be solved by $N \times N$ simultaneous equations. A signal spectrum corrected with the above procedure is shown in Fig. 15. The main part of the spectrum is accurately retrieved. Some oscillatory features in the peripheral spectral regions may be caused by the imperfection of the $3 \mathrm{~dB}$ couplers and differential attenuation in longer asymmetrical MZIs. Correction of these imperfections will be reported in the future.

\section{SUMMARY}

Four kinds of silicon photonics WDM filters have been investigated in detail. It is made clear that (a) improvement in core width and thickness uniformity and (b) size reduction to make the device size small are strongly required in order to achieve good crosstalk characteristics $(<-20 \mathrm{~dB})$.

We have demonstrated a novel planar waveguide spectrometer implemented in silica PLC. The signal spectrum was successfully retrieved by correcting the MZI output data with the phase error values. Miniature spectrometers will be important for daily health care and environmental sensing applications since they are compact in size and potentially very low cost (disposable).

\section{REFERENCES}

1. A. Yariv, Y. Xu, R. K. Lee, and A. Scherer, "Coupledresonator optical waveguide: a proposal and analysis," Opt. Lett. 24, 711-713 (1999).

2. K. Jinguji and M. Oguma, "Optical half-band filters," IEEE J. Lightwave Technol. 18, 252-259 (2000).

3. K. Okamoto, Fundamentals of Optical Waveguides, 2nd ed. (Elsevier, New York, USA, 2006), Chapter 9.

4. Z. Shi and S. He, "A three-focal-point method for the optimal design of a flat-top planar waveguide demultiplexer," IEEE J. Select. Topics Quantum Electron. 8, 1179-1185 (2002).
5. K. Takada, Y. Inoue, H. Yamada, and M. Horiguchi, "Measurement of phase error distributions in silica-based arrayed-waveguide grating multiplexers by using Fourier transform spectroscopy," Electron. Lett. 30, 1671-1672 (1994).

6. K. Okamoto, "Evolution of planar waveguide devices: communication and sensing applications," in Proc. ECIO 2010 (Cambridge, UK, April 2010), paper FrA.

7. J. J. He, B. Lamontagne, A. Delage, L. Erickson, M. Davies, and E. S. Koteles, "Monolithic integrated wavelength demultiplexer based on a waveguide rowland circle grating in InGaAsP/InP," IEEE J. Lightwave Technol. 16, 631-638 (1998).

8. F. Xia, L. Sekaric, and Y. Vlasov, "Ultracompact optical buffers on a silicon chip," Nature Photonics 1, 65-71 (2007).

9. M. Romagnoli, "Silicon on insulator based integrated flexible optical components," in Proc. ECOC '07 Workshop (Berlin, Germany, Sep. 2007).

10. D. Van Thourhout and W. Bogaerts, "Silicon photonics," in Proc. OFC '10 (San Diego, CA, USA, Mar. 2010), paper OtuB5.

11. W. Bogaerts, P. Dumon, D. Van Thourhout, D. Taillaert, P. Jaenen, J. Wouters, S. Beckx, V. Wiaux, and R. G. Baets, "Compact wavelength-selective functions in siliconon-insulator photonic wires," IEEE J. Select. Topics Quantum Electron. 12, 1394-1401 (2006).

12. D. J. Kim, J. M. Lee, J. H. Song, J. Pyo, and G. Kim, "Crosstalk reduction in a shallow-etched silicon nanowire AWG," IEEE Photon. Technol. Lett. 20, 1615-1617 (2008).

13. Q. Fang, T. Y. Liow, J. F. Song, K. W. Ang, M. B. Yu, G. Q. Lo, and D. L. Kwong, "WDM multi-channel silicon photonic receiver with 320 Gbps data transmission capability," Opt. Express 18, 5106-5113 (2010).

14. J. Brouckaert, W. Bogaerts, P. Dumon, D. Van Thourhout, and R. Baets, "Planar concave grating demultiplexer fabricated on a nanophotonic silicon-on-insulator platform," IEEE J. Lightwave Technol. 25, 1269-1275 (2007).

15. W. Bogaerts, S. K. Selvaraja, P. Dumon, J. Brouckaert, K. D. Vos, D. Van Thourhout, and R. Baets, "Silicon-oninsulator spectral filters fabricated with CMOS technology," IEEE J. Select. Topics Quantum Electron. 16, 33-44 (2010).

16. F. Horst, W. M. J. Green, B. J. Offrein, and Y. A. Vlasov, "Silicon photonic WDM devices: simulation, design and implementation," Photonics North 2009, Proc. SPIE 7386, 73862 L1-L9 (2009).

17. J. M. Harlander, F. L. Roesler, J. G. Cardon, C. R. Englert, and R. R. Conway, "SHIMMER: a spatial heterodyne spectrometer for remote sensing of Earth's middle atmosphere," Appl. Opt. 41, 1343-1352 (2002).

18. M. Florjańczyk, P. Cheben, S. Janz, A. Scott, B. Solheim, and $\mathrm{D} . \mathrm{X}$. Xu, "Multiaperture planar waveguide spectrometer formed by arrayed Mach-Zehnder interferometers," Opt. Express 15, 18176-18189 (2007).

19. D. Noordegraaf, P. M. W. Skovgaard, M. D. Maack, J. B. Hawthorn, R. Haynes, and J. Lagsgaad, "Multi-mode to single-mode conversion in a 61 port photonic lantern," Opt. Express 18, 4673-4678 (2010).

20. M. Florjańczyk, P. Cheben, S. Janz, B. Lamontagne, J. Lapointe, A. Scott, B. Solheim, and D. X. Xu, "Development of slab waveguide spatial heterodyne spectrometer for remote sensing," Proc. SPIE 7394, 75940 R1-R9 (2010). 
21. K. Okamoto, H. Aoyagi, and K. Takada, "Fabrication of Fourier-transform, integrated-optic spatial heterodyne spectrometer on silica-based planar waveguide," Opt. Lett. 35, 2103-2105 (2010)

22. T. Goh, S. Suzuki, and A. Sugita, "Estimation of waveguide phase error in silica-based waveguides," IEEE J. Lightwave Technol. 15, 2107-2113 (1997).

23. T. Kominato, T. Kitoh, K. Katoh, Y. Hibino, and M. Yasu, "Loss characteristics of intersecting silica-based waveguides," in Proc. Optoelectronics Conf. OEC' 92 (Makuhari, Japan, Jul. 1992), pp. 138-139.

24. K. Takada, T. Tanaka, M. Abe, T. Yanagisawa, M. Ishii, and K. Okamoto, "Beam-adjustment-free crosstalk reduction in $10 \mathrm{GHz}$-spaced arrayed-waveguide grating via photosensitivity under UV laser irradiation through metal mask," Electron. Lett. 36, 60-61 (2000).

25. G. Fan and Q. H. Liu, "Fast Fourier transform for discontinuous functions," IEEE Trans. on Antennas and Propagation 52, 461-465 (2004). 